\title{
SERVICIOS ESTUDIANTILES EN LA UNIVERSIDAD DIEGO PORTALES: UNA APUESTA POR LA CALIDAD
}

Roberto Vega* 


\section{RESUMEN}

El autor aborda de manera sintética el tema de los servicios estudiantiles y cómo ellos son parte relevante de la formación que se entrega a los alumnos en el nivel universitario. Hace además referencia a la importancia que adquiere la planificación estratégica en el logro de objetivos y resultados. Relacionado con lo anterior, explica el modelo que ha seguido la Universidad Diego Portales en el ámbito de asuntos estudiantiles para los procesos de acreditación internacional, pasando por una descripción del modelo de autoestudio. Finalmente, expone de manera general el modelo de planificación anual y control de gestión que utilizan las diversas unidades que componen la Dirección de Asuntos Estudiantiles.

\section{ABSTRACT}

The author briefly addresses the issue of student services and how these are an important part of the education provided to students at university level. He also refers to the importance acquired by strategic planning in achieving objectives and results. In connection with the above, he explains the model followed by the Universidad Diego Portales in matters related to student affairs for international accreditation, including a description of the model of individual studying. Finally, the author presents, in general terms, the model of annual planning and management control used by the different units that form the Department of Student Affairs. 


\section{SERVICIOS ESTUDIANTILES EN LA UNIVERSIDAD DIEGO PORTALES: UNA APUESTA POR LA CALIDAD}

Las universidades privadas en su conjunto, desde su fundación en 1982 a la fecha, han debido sortear situaciones complejas en pro de su crecimiento y desarrollo institucional. Su búsqueda se ha centrado, en primer término, en la legitimación de su calidad académica ante la sociedad, y luego, en demostrar que es posible entregar una educación de nivel superior más allá de las fronteras estatales.

El tema del desarrollo y crecimiento experimentado por el sistema universitario privado podría ser objeto de una interesante investigación, que no se limitara sólo a la medición de variables como el crecimiento de matrícula, infraestructura y otros registros cuantitativos de los que regularmente dan cuenta las monografías, las investigaciones específicas y rankings de universidades. Sería menester que analizara además los resultados centrados en la calidad de los alumnos formados, los aportes en la construcción de mallas curriculares, la nueva oferta de carreras, la aplicación de nuevas metodologías de enseñanza, proyectos de investigación y, en definitiva, aportes a la solución de los problemas de la sociedad globalmente considerada. Los veinte años transcurridos ameritan profundizar en la dinámica de la enseñanza superior privada y su impacto en el mundo social.

Sirvan estos comentarios de carácter general para introducir un tema específico: la importancia que posee una unidad de servicios estudiantiles al interior de una universidad privada. No cabe duda de que los fenómenos de regulación externos a una institución condicionan su desarrollo en lo interno, particularmente cuando su actividad en los primeros años está orientada a obtener la autonomía plena. Con esto deseamos decir que, obviamente, la actividad de la academia es lo primero y más importante y que los servicios estudiantiles -en una primera etapa- son entendidos como parte de una "formación integral", pero que también son generalmente 
percibidos más como un gasto que como una actividad secundaria, posible de postergar en el tiempo.

En lo personal me ha correspondido vivir la experiencia de creación y direccionamiento de unidades de asuntos estudiantiles desde una perspectiva tradicional (Universidad de Santiago) y privada (Universidad Diego Portales). En el presente artículo no intentaré hacer un estudio comparativo, porque resulta inoficioso y metodológicamente complejo de realizar debido a cuestiones que van más allá del tamaño e historia de ambas instituciones.

Al respecto, sólo diremos que existe una diferencia de vitalidad y fluidez en la concreción de objetivos y la realización de actividades a favor del sistema privado. El fenómeno del impacto que el tamaño de las instituciones de educación superior tiene en la efectividad y eficiencia para la concreción de objetivos, es un tema que la modernidad ha puesto de manifiesto. En este ámbito se insertan cuestiones tan básicas como presupuesto disponible, recursos humanos y capacidad tecnológica instalada.

En el desarrollo de este artículo daremos cuenta de las áreas que componen la Dirección General de Asuntos Estudiantiles de la Universidad Diego Portales.

\section{DESCRIPCIÓN GENERAL}

En sus orígenes la Dirección de Asuntos Estudiantiles estuvo compuesta por tres unidades: Bienestar Estudiantil, Deportes, Cultura y Comunicación, todas dependientes de la Secretaría General, para mantener una vinculación con la administración central. En la actualidad, la Dirección General de Asuntos Estudiantiles, depende de la Vicerrectoría de Comunicación, por estimarse que en todas las acciones que se desarrollan en beneficio de los alumnos existe una acción dialéctica, en la que aparece de por medio la comunicación de marca.

Con anterioridad a esta nueva dependencia de la Dirección de Asuntos Estudiantiles, ésta poseyó el carácter de Vicerrectoría (1999 
y 2000), luego durante el año 2001 estuvo bajo la dependencia de la Vicerrectoría Académica. En cuanto a su actual estructura y dependencia véase Anexo I.

Durante los primeros años, el desarrollo de programas se caracterizó por su volumen e impacto, pero no necesariamente por su sistematización. El acento estuvo puesto en la construcción de un ambiente universitario tendiente a una formación amplia y pluralista, vinculando a los alumnos con la modernidad y con los problemas de la sociedad. Asimismo, se buscaba mantener vigente la idea de dotar a la Universidad de buenos académicos y apostar a los más altos niveles de excelencia en el rendimiento de los alumnos.

Otro hecho que marca la tendencia y orientación de la Universidad Diego Portales desde sus orígenes es la importancia asignada a las organizaciones estudiantiles, estableciendo mecanismos que permitan su vinculación con los cuerpos colegiados de la institución. Por ello, los presidentes de los centros de alumnos tienen representación con voz y voto en los Consejos Académicos de Facultad y el presidente de la Federación de Estudiantes (FEDEP) posee representación en el Consejo Académico de la Universidad.

En 1997 la Universidad pone en marcha un programa de estímulo a la creación: los grupos de interés, concebidos como instancias de participación y expresión de las motivaciones de los alumnos en su más amplia cobertura (juegos de rol, Parapsicología, Ufología, rodeo, pastoral universitaria, misiones católicas, etcétera).

El año 1998 se crea la corporación de ex alumnos, con el objeto de mantener vínculos entre los alumnos egresados, por el aporte que ellos representan desde el punto de vista de su visión externa de la Universidad.

\section{MEDICIÓN DE LA CALIDAD}

Es posible medir el perfeccionamiento de los procesos educativos y de servicios que se entregan a los estudiantes a través de la acreditación, indudablemente, uno de los fenómenos más nuevos al interior de la 
educación chilena. En el ámbito nacional, si bien la discusión ha sido intensa, ésta se ha centrado -lastimosamente- en la definición del modo en que se debe realizar (acreditación institucional o por programas) y en quién debe realizarla (entidad acreditadora estatal o privada).

Nuestra Universidad ha entendido la importancia que la acreditación tiene como método de autorregulación, de fortalecimiento y respaldo a la calidad e integridad de la educación y sus componentes. Una medida del interés de la Universidad por la calidad de la educación superior y del compromiso que tiene por procurar y alcanzar la excelencia a través de sus esfuerzos, es el grado en el cual cada institución acepta y cumple los requisitos inherentes al proceso.

Es indudable que el principal indicador de la calidad de una institución es la habilidad con la que ésta define sus objetivos. A partir de este precepto, la planificación de actividades y tareas que desarrollan las diversas unidades que integran la Vicerrectoría de Comunicación y por ende la Dirección General de Asuntos Estudiantiles, se formula con base en una definición de públicos externos e internos, en el entendido de que la "marca" Universidad Diego Portales, se comunica a esos públicos a través de canales, fórmulas y acciones, para el cumplimiento de objetivos estratégicos.

Así entonces, una vez definidos los públicos se establece una estrategia y, respecto de ésta, se fijan tareas específicas que serán implementadas por cada una de las unidades mediante la ejecución de acciones y dentro de unos plazos para el cumplimiento de las mismas.

La metodología aquí señalada permite un notable control y autoevaluación de la gestión, como también la focalización de los recursos (humanos y materiales) con un sentido de realidad. La improvisación -siempre válida en procesos humanos no infalibles- se intenta reducir a su mínima expresión.

En las funciones y procesos más relevantes se promueve la idea de elaborar documentos e informes de detalle, que permitan el perfeccionamiento de procesos posteriores; por ejemplo, la Dirección de Admisión debe emitir un informe anual de orden cuantitativo y 
cualitativo, construyendo así bases de datos históricos, que proporcionan a la institución un conocimiento exacto de su posición en el mercado educacional.

Otras tareas de nivel medio deben ser informadas con el máximo rigor, evaluando fortalezas y debilidades de la acción emprendida, así como de la definición de proyecciones futuras en la misma actividad. Sin lugar a dudas, se pueden citar como ejemplos exitosos de esta forma de proceder las siguientes actividades: Escuela de Invierno, entendida ésta como una actividad de perfeccionamiento de los profesores de enseñanza básica y media, la que ha logrado conciliar el compromiso de profesores de todas las facultades de la Universidad, y el apoyo de entes externos, tan relevantes como el Ministerio de Educación y el Centro de Perfeccionamiento, Experimentación e Investigaciones Pedagógicas. La actividad, que ya tiene once años de vida, ha sido perfeccionada a través de la aplicación de diversos instrumentos de medición, buscando nuevas fórmulas creativas de enseñanza que no se divorcien de las necesidades reales de profesores y profesionales del área de la educación en general.

Otra actividad que responde con igual rigor es la organización de los torneos interescolares de debate (quinta versión en el año 2002, con participación de 56 colegios, 76 equipos de debate y aproximadamente 550 alumnos vinculados a la actividad), que cada vez han verificado la presencia de una mayor cantidad de alumnos mejor dotados en el arte de la retórica, la oratoria y la argumentación.

Lo óptimo es que una institución, al evaluar sus objetivos en materia estudiantil, deba conocer y comprender a las poblaciones de alumnos actuales y futuras. Esto incluye, en un nivel superior, creencias, actitudes, valores, intereses, habilidades, conocimiento cultural y otros aspectos del desarrollo psicológico y social del estudiante. El conocimiento de las características de los estudiantes influye y puede guiar los procesos de enseñanza y aprendizaje, el ambiente institucional, los programas ofrecidos y los servicios proporcionados. Cuando los servicios al estudiante están correctamente diseñados y se corresponden con la misión y objetivos de la institución, refuerzan y extienden la influencia de la institución más allá del aula. Como resultado, estos servicios se tornan parte integral del proceso educativo. 
En virtud de las ventajas que reportan los procesos de acreditación, la Universidad Diego Portales, en su calidad de miembrofundador de la Red Latinoamericana de Cooperación Universitaria (RLCU), se acogió voluntariamente a un proceso de acreditación institucional el año 1998, a partir de un autoestudio. En ese proceso de autoestudio se construyó, en el nivel institucional, una tabla de indicadores por dimensión y según criterios mínimos a cumplir; a cada uno de ellos se asignó otra tabla que permitió establecer dimensión, característica e indicadores y, en relación con estos últimos, se fijaron instrumentos de medición y audiencias o fuentes vinculadas al cumplimiento de ese indicador.

La mayor dificultad observada en este autoestudio fue probablemente la construcción de instrumentos de medición que fueran más allá de los cuestionarios, entrevistas, observación, archivos internos, informes, formularios, encuestas, reuniones, etc., toda vez que existe la tendencia de orientar los resultados a la construcción de tablas y gráficos de orden cuantitativo, descuidando una medición más fina en lo cualitativo.

Finalmente, antes de iniciar la descripción de las diversas unidades y programas que forman parte de la Dirección General de Asuntos Estudiantiles de la Universidad Diego Portales, debo abordar un tema no menor y que constituye un freno al desarrollo de las actividades entregadas como servicio. Me refiero a la falta de valor-crédito (y obligatoriedad) de las acciones que se desarrollan con una clara orientación académica (talleres, seminarios, cursos) en el ámbito del deporte, la cultura y en el caso nuestro en el debate, pero que finalmente no poseen ese carácter.

Es decir, la amplia oferta de cursos en el ámbito de los servicios regularmente no posee el valor de crédito ni ningún otro valor en el currículum de los alumnos. Con ello se provocan situaciones que atentan contra el logro de los objetivos del área de servicios estudiantiles: $a$ ) los alumnos tienden a abandonar sus cursos formativos en deporte y cultura, en período de exámenes; $b$ ) los estudiantes estiman que estos cursos, por su carácter, deben ser más fáciles o menos exigentes; $c$ ) lo que es más grave, se tiende a perder el esfuerzo humano y profesional invertido en ellos. 
La Universidad Diego Portales ha abordado este tema, en una primera etapa, haciendo que los alumnos puedan sumar la nota obtenida en estos cursos a su promedio general de carrera.

Sin lugar a dudas, éste es uno de los temas más complejos para las universidades que comienzan a avanzar en la definición de una unidad de asuntos estudiantiles, toda vez que se ven enfrentadas a estructuras curriculares poco flexibles, que no dan cabida a una oferta de cursos distinta de los de especialidad.

\section{DIRECCIÓN DE PROGRAMAS ESTUDIANTILES}

En primer lugar nos referiremos a la Dirección de Programas Estudiantiles, creada el año 2000 para asesorar las actividades que desarrollan las organizaciones estudiantiles y canalizar las necesidades e inquietudes propias de los alumnos. También busca promover y colaborar en la creación y desarrollo de grupos de interés, que permitan expresar y plasmar las inquietudes de los alumnos a través de actividades y proyectos culturales, recreativos, deportivos y sociales.

Otra función destacable es la de promover la participación en el Programa "Adopta un Herman@”, en forma conjunta con la Fundación Nacional para la Superación Nacional de la Pobreza.

En materia de extensión coorganiza, con la Unidad de Admisión, eventos dirigidos a establecimientos educacionales (Campeonato Interescolar de Vóleibol, Campeonato Interescolar de Babyfútbol, Campeonato Interescolar de Rugby).

\section{DEPARTAMENTO DE BIENESTAR ESTUDIANTIL Y ATENCIÓN PSICOLÓGICA}

Desde sus inicios la Universidad incluyó en su estructura organizacional la Unidad de Bienestar Estudiantil, asimilándose a las universidades de mayor tradición en el país y constituyéndose en pionera en esta materia respecto de las otras universidades privadas. 
A contar de 1984 se implementó un programa de becas de arancel y con el transcurso del tiempo se incorporaron nuevos beneficios acordes con las necesidades del alumnado.

Entre sus principales actividades esta unidad presta un servicio de apoyo a alumnos y apoderados con situaciones conflictivas en el ámbito socioeconómico y académico-vocacional, que interfieren el normal desarrollo de los estudios.

El Departamento de Bienestar Estudiantil debe mantener una coordinación permanente con las instituciones extrauniversitarias mediante normativas propias, que favorecen a alumnos de nuestra Universidad, tales como: becas externas; Beca Presidente de la República; Beca Juan Gómez Millas; Beca Para Hijos de Profesionales de la Educación; Beca para Estudiantes Destacados que Ingresan a Pedagogía y Beca Indígena.

Además, este departamento maneja otros tipos diferentes de becas, como las becas de excelencia académica, que consisten en la rebaja del arancel anual por el primer año de la carrera a favor de los alumnos que ingresan con un puntaje de Prueba de Aptitud Académica (PAA) igual o superior a 700 puntos y obtienen el primer lugar de selección en la respectiva carrera. Las becas de mérito, que se entregan a los alumnos egresados de la enseñanza media fiscal, que acrediten un puntaje igual o superior a 680 puntos y que obtengan el primer lugar de selección en la respectiva carrera. También se contempla un programa de becas alimenticias, el que incluye además entre sus funciones internas inmediatas la distribución de los pases escolares que asignan el Ministerio de Educación y el Consejo de Transporte.

Con el objeto de cubrir la matrícula, arancel y costos de titulación, se estableció un sistema de seguro universitario en el caso de fallecimiento del padre o sostenedor del alumno, hasta el término de la carrera. Además, se implementó un seguro de cesantía y hospitalización para los alumnos vespertinos.

En septiembre de1989 se crea el Servicio de Atención Psicológica, el que se orienta a las demandas de salud mental de los alumnos de la Universidad. Con el tiempo este servicio ha ido ampliando su capacidad 
de atender las necesidades de los alumnos, llegando a cerca de 650 horas de atención psicológica el año 2001.

Es importante mencionar también que el Servicio de Atención Psicológica se ha orientado tanto al trabajo individual como grupal con los alumnos, así como también al ámbito de la atención y de la prevención. Ejemplo de ello fue la implementación de un Programa Preventivo de Drogas y Alcohol, llevado a cabo en la Facultad de Ciencias de la Comunicación y la Escuela de Ingeniería Comercial.

Entre otras funciones el Servicio de Atención Psicológica debe:

- Hacer una evaluación y diagnóstico psicológicos, por iniciativa propia de los alumnos o derivados por alguna instancia académica de la Universidad.

- Realizar intervenciones psicológicas, las que se desarrollan mediante un proceso de entrevistas de evaluación y por medio del tratamiento propiamente tal.

- Realizar procesos de derivación, consistentes en evaluar y hacer la interconsulta pertinente del alumno.

\section{DEPORTES}

Las directrices que orientan el quehacer de esta unidad son promover la participación de las selecciones deportivas de la Universidad, tanto en el nivel nacional como internacional, así como fomentar el diseño de mecanismos que permitan la amplia práctica deportiva de los alumnos y la incorporación de estudiantes deportistas destacados en el proceso de admisión a la Universidad y su posterior supervisión.

Dada la importancia que se asigna al deporte, la Universidad construyó en el período 2000-2001 un moderno edificio climatizado, de más de 6 mil metros cuadrados distribuidos en seis niveles, para la práctica de diversos deportes como: babyfútbol, vóleibol, básquetbol, tenis, raquetbol. Complementan esta infraestructura tres salas de máquinas con equipamiento de última generación, dos saunas y dos multisalas. 


\section{CULTURA}

El desarrollo educacional busca propiciar un encuentro con la cultura y el arte acorde con los intereses de los alumnos, permitiendo una participación basada en la diversidad, que favorezca un espíritu crítico y pluralista. En el marco de este objetivo se han planteado acciones que ofrecen diversas alternativas a toda la comunidad portaliana en las áreas de: talleres; artes visuales (exposiciones, instalaciones, acciones de arte, etcétera); música (presentaciones de Grupos UDP y externos); teatro (presentaciones de Grupos UDP y externos); ciclos de cine (propuestas conectadas con escuelas y centros de alumnos) y otros eventos (conferencias, seminarios y producciones interdisciplinarias). Esta área lleva diez años de funcionamiento sistemático.

Durante todo el año, el Departamento de Cultura mantiene un nutrido programa de actividades que son permanentemente evaluadas; para ello, cada semestre se realiza un seguimiento y estudio estadístico a través de instrumentos de evaluación (encuestas de ingreso y egreso de cursos o talleres), con el fin de captar los intereses reales de los estudiantes en esta área y orientar la oferta de cursos. A fin de año se entrega un diploma para acreditar la participación de los alumnos que cumplieron con los objetivos planteados en cada taller.

Existen tres grupos estables (música, teatro y fotografía), orientados al desarrollo de sensibilidades por medio del conocimiento de técnicas de expresión, que presentan su trabajo dentro y fuera del espacio universitario a lo largo del año. El grupo de teatro (nueve años de existencia) cuenta con premios en encuentros y festivales teatrales en Santiago; el grupo de música (creado en abril de 2000), obtuvo una distinción especial en el ler Encuentro Musical Universidad Andrés Bello; un año después, se formó el grupo de fotografía, que destacó con una notable exposición en el Goethe Institut.

Como una forma de vincular a los alumnos con las artes visuales se organizan exposiciones en las distintas facultades, que abarcan grabado, pintura, fotografía, escultura e instalaciones, privilegiando la presencia de artistas emergentes nacionales. 


\section{SOCIEDAD DE DEBATE}

Creada en 1994, la Sociedad de Debate de la Universidad Diego Portales fomenta la teoría y la práctica del debate e impulsa el desarrollo de habilidades comunicativas. A través de torneos competitivos, docencia, publicaciones, charlas, capacitación y extensión, busca promover la cultura del debate en nuestro país. Actualmente recibe apoyo de la Sociedad de Debates Filodémica de la Universidad de Georgetown y del World Debate Institute de la Universidad de Vermont.

En forma progresiva el debate se ha logrado consolidar como una herramienta valiosa y coherente con los principios de la Universidad. En una primera instancia esta unidad enfocaba su labor principalmente a la enseñanza y práctica competitiva del debate al interior de la Universidad; hoy se ha transformado en una entidad de extensión, docencia y capacitación.

Entre las principales actividades está el Torneo Interescolar de Debate Competitivo, el más antiguo que se realiza en el país, que se lleva a cabo desde 1998 y reúne a los colegios de la Región Metropolitana. Desde el año 2000 se han sumado iniciativas regionales propiciadas por el Campus Sur-Austral (Temuco) de la Universidad Diego Portales, existiendo en la actualidad torneos en Temuco, Puerto Montt, Osorno y Coyhaique. El año 2001, en tanto, se realizó el Primer Torneo Hispanoamericano de Debate, que contó con la presencia de representantes de ocho universidades de Latinoamérica, España y un equipo hispanoparlante de EE.UU. La segunda versión se realizó este año en Puerto Rico.

En materia de docencia se privilegia la realización de talleres y seminarios de expresión oral, argumentación y persuasión, dirigidos a los estudiantes de la Universidad y al público en general.

El año 2002 se crea la Red Nacional de Debate (http:// www.renade.cl) con el objeto de difundir el debate competitivo y la argumentación a través de Internet.

También la Sociedad de Debate ha puesto en marcha un programa de capacitación docente a lo largo de todo el país, dictando cursos de debate competitivo y expresión oral, a través del beneficio SENCE. 
En lo que respecta a publicaciones, existen dos medios: la revista Versus, que recoge artículos y reportajes de temas de controversia, y la Colección Argumento, iniciada con el libro Métodos de razonamiento para el debate, del profesor José Julio León.

Uno de los principales logros del programa de debate ha sido la puesta en marcha del Proyecto TIMD (Torneo Interescolar Metropolitano de Debate), desarrollado con financiamiento del Ministerio de Educación, que implementará capacitaciones y torneos de debate en siete zonas provinciales de la Región Metropolitana, dirigido a profesores y alumnos de liceos de zonas socialmente vulnerables.

En materia de extensión se ha dado vida al primer "Informe de Calidad del Debate", que, con un carácter trimestral, pretende dar cuenta de la calidad del debate presente en la agenda pública, mediante el análisis en profundidad de una polémica contingente en los medios de comunicación y la entrega de pautas de análisis argumental.

\section{DIRECCIÓN DE INTERCAMBIO ESTUDIANTIL INTERNACIONAL}

Los procesos de globalización han motivado a la institución a establecer, desarrollar y mantener convenios de intercambio estudiantil con universidades extranjeras de América, Europa y Asia. Para ello se ha creado la Dirección de Intercambio Estudiantil Internacional, que tiene por objeto asesorar a estudiantes y profesionales egresados de UDP, interesados en estudios de postgrados en universidades en el extranjero.

Se vincula con las facultades a través del Comité de Intercambio Estudiantil Internacional, cuyos integrantes (representantes de todas las facultades de la Universidad), se reúnen mensualmente para difundir y administrar los programas de intercambios estudiantiles internacionales.

Algunas de las acciones desarrolladas por la Dirección de Intercambios Estudiantiles Internacionales son las siguientes:

a) Vigencia de más de 80 convenios con universidades extranjeras; 
b) Programas de inglés intensivo en Boston University, University of Delaware, University of New Orleans y Tulane University;

c) Página web con información de convenios, becas, servicios para estudiantes extranjeros, etcétera;

d) Centro de información documental de universidades con las que UDP tiene convenios.

En tanto, los alumnos extranjeros - provenientes de universidades de América y Europa- que concurren semestralmente a UDP cuentan con un sólido sistema de apoyo integrado por:

a) Apoyo institucional a través de la Dirección de Intercambios Estudiantiles Internacionales;

b) Asignación de profesores tutores en sus respectivas escuelas;

c) Sistema de "apadrinamiento" de parte de estudiantes de la U n i versidad.

El sistema de intercambios estudiantiles de UDP ha logrado crecer de manera sólida y eficiente, dando respuestas adecuadas a las necesidades de los estudiantes de la Universidad y de los estudiantes extranjeros que semestralmente participan en intercambios de reciprocidad. Cada año se ha logrado una movilidad de aproximadamente 250 estudiantes chilenos y extranjeros, que han enriquecido sus vidas con experiencias internacionales.

\section{DIRECCIÓN DE ADMISIÓN}

Es la unidad responsable de los procesos de admisión correspondientes a los alumnos de pregrado. Formula estrategias de difusión y campañas de promoción de las carreras, permitiendo así la creación de nuevas alternativas comunicacionales. Desde sus orígenes, la Unidad de Admisión ha sido concebida como un área vinculada a servicios estudiantiles.

Algunas de sus funciones son:

- Coordinar y supervisar los procesos de admisión regular de los alumnos que postulan a las carreras de pregrado; 
- Formar y capacitar a los equipos de admisión para cada proceso, así como a los equipos de charlistas y promotores de ferias vocacionales (preferencialemente alumnos de la misma Universidad);

- Coordinar con la Dirección de Planificación y Desarrollo la realización de estudios que permitan mantener bases de datos actualizadas sobre procesos de admisión nacional, orientadores, establecimientos educacionales y similares.

La planificación coherente, sistemática y unitaria de esta Dirección se ha traducido en la generación permanente de nuevas fórmulas de difusión.

\section{UNIDAD DE MEDIOS DE COMUNICACIÓN}

Tiene como objetivo desarrollar la comunicación interna y externa, potenciando la percepción de la Universidad Diego Portales entre sus diversos públicos, con la perspectiva de comunicarles el quehacer universitario, posicionando a la UDP como una institución de educación superior de excelencia académica y con vocación pública.

Para esto se desarrollan permanentemente iniciativas orientadas a mostrar lo que se realiza en la UDP, tanto en prensa como en radio y televisión. En forma paralela, existe un medio de comunicación interna: "El Portaliano" (que se difunde utilizando como soportes una edición en papel y otra en un sitio web a través de Internet), publicación mensual con un tiraje de 4.000 ejemplares, que se distribuye tanto a la comunidad portaliana como a público externo.

Entre las principales actividades de la Unidad de Medios de Comunicación, se encuentra la cobertura periodística de todas las actividades que se realizan al interior de la Universidad. Este año se ha puesto el acento en desarrollar una serie de actividades destinadas a promover a los académicos de la UDP en los medios de comunicación. 


\section{UNIDAD DE RELACIONES PÚBLICAS Y PROTOCOLO}

La Unidad de RR.PP. y Protocolo tiene un lugar importante en la realización de cada una de las ceremonias y eventos institucionales que las distintas unidades académicas organizan periódicamente. Su objetivo es organizar, producir y apoyar los distintos actos oficiales de la Universidad, con el ceremonial y protocolo pertinentes. En el ámbito de las relaciones públicas internas existe un trabajo permanente con y hacia los distintos públicos con los cuales existen vínculos.

En lo que respecta a las relaciones públicas externas, destaca la presencia sostenida de la Universidad en eventos nacionales e internacionales.

En su origen, esta unidad recibió el nombre de "Asuntos Corporativos", que dependía de la Dirección de Asuntos Estudiantiles y se concentraba en la realización, producción y montaje de cada una de estas actividades. En 1996 se creó la Unidad de Relaciones Públicas en Casa Central, que se encarga de llevar un estricto control y supervisión de los actos atingentes a la Rectoría y demás instancias superiores. La coordinación entre la Casa Central y la Dirección de Asuntos Estudiantiles es clave para la realización y desarrollo de cada una de las ceremonias que se organizan al interior de nuestra Universidad.

Ante un escenario especialmente competitivo y exigente en términos corporativos, la Universidad Diego Portales se ve en la necesidad de crear la Dirección de Relaciones Públicas y Protocolo, que vela por el correcto uso de la imagen corporativa de la institución y la organización de las actividades oficiales de nuestra casa de estudios superiores. 


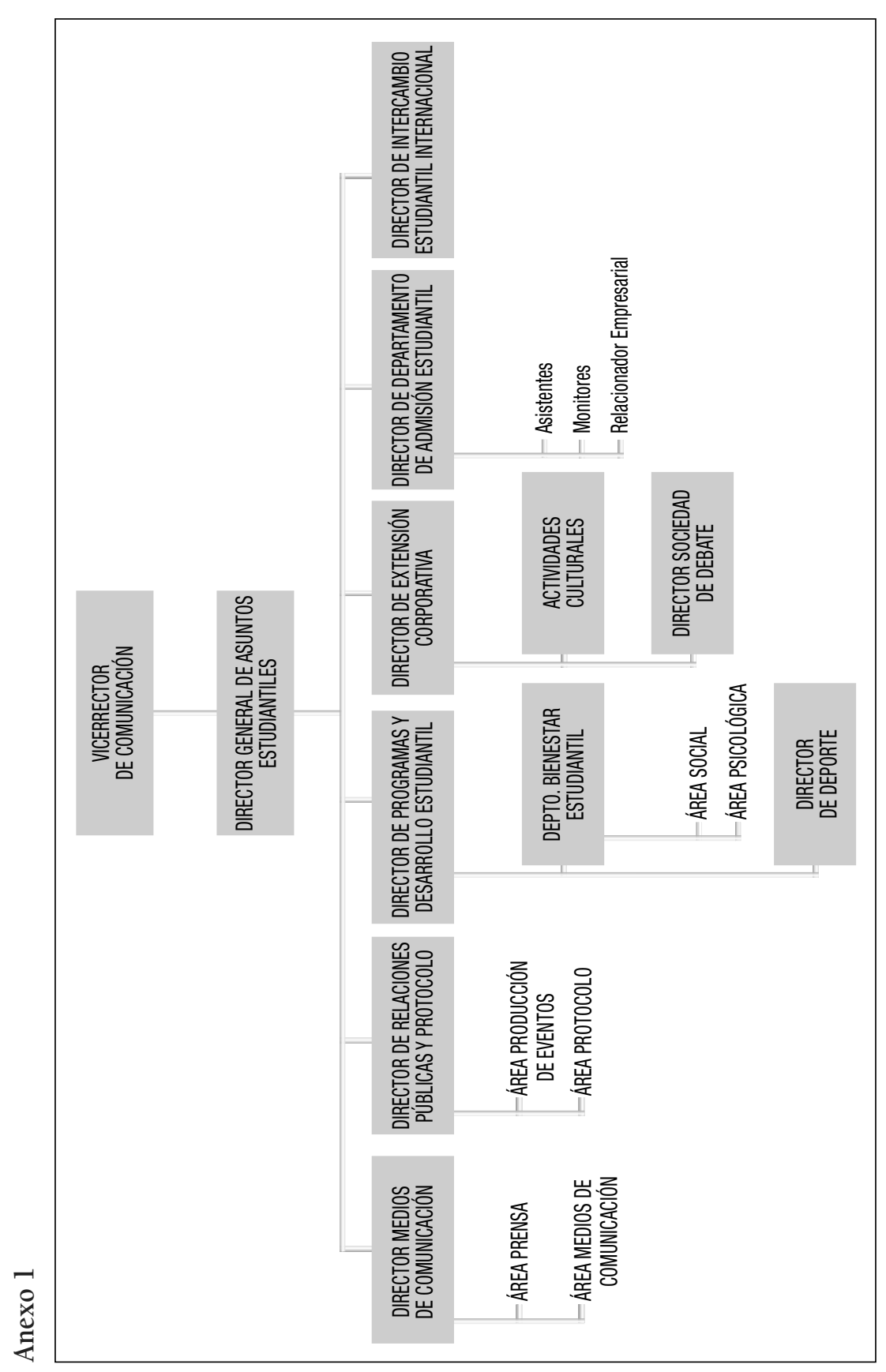

